\title{
ON THE PRECISION OF THE ISOTROPIC CAVALIERI DESIGN
}

\author{
Javier GonzÁlez-Villa ${ }^{\bowtie}$, Marcos Cruz And Luis M. CRuZ-Orive \\ Department of Mathematics, Statistics and Computation, Faculty of Sciences, University of Cantabria, Avda. \\ Los Castros 48, E-39005 Santander, Spain. \\ e-mail: javier.gonzalezv@alumnos.unican.es,marcos.cruz@unican.es, luis.cruz@unican.es \\ (Received May 15, 2018; revised September 21, 2018; accepted September 21, 2018)
}

\begin{abstract}
The isotropic Cavalieri design is based on a isotropically oriented set of parallel systematic sections a constant distance apart. Its advantage over the ordinary Cavalieri design is twofold - first, besides volume it allows the unbiased estimation of surface area, and second, the error variance predictor for the volume estimator is much simpler, involving only the surface area of the object, and the distance between sections. In an earlier paper, the two hemispheres of a rat brain were arranged perpendicular to each other before sectioning, aiming at reducing the error variance with respect to other arrangements (such as the aligned one) by exploiting an intuitively plausible antithetic effect. Because the total surface area of the objects is unchanged under any arrangements, however, the error variance predictor for the volume estimator does not depend on object shape, which looks intriguing. Using reconstructions of the mentioned hemispheres, we dilucidate the aparent paradox by means of automatic Monte Carlo replications of the relevant volume estimates under the antithetic and the aligned arrangements.
\end{abstract}

Keywords: brain, image analysis, isotropic Cavalieri, stereology, variance prediction, volume.

\section{INTRODUCTION}

Consider a fixed, compact set $Y \subset \mathbb{R}^{3}$, (e.g. the rat brain reconstruction studied here), not necessarily connected, of unknown volume $V$, with piecewise smooth boundary $\partial Y$ of area $S$. To estimate $V$, the isotropic Cavalieri design (ICav) is based on parallel, systematic sectioning planes a constant distance $T$ apart, along a normal sampling axis whose direction $u \in \mathbb{S}_{+}^{2}$ is isotropic random on the unit hemisphere (Cruz-Orive et al., 2010). Thus, the only difference between the ICav and the ordinary Cavalieri design is that, in the former, the direction of the sampling axis is isotropic. The ICav is not necessary to estimate $V$ because the Cavalieri design is unbiased for any sampling direction. The ICav was used in the latter paper, however, because it allows the simultaneous unbiased estimation of $S$.

Compared with the error variance predictors for the volume estimator under the ordinary Cavalieri design (Gundersen and Jensen, 1987; Kiêu et al., 1999; García-Fiñana and Cruz-Orive, 2004; CruzOrive, 2006), the predictor for the ICav is simpler: it depends on $S$ and $T$ only, see Eq. 10 below. In Cruz-Orive et al. (2010) the subset $Y$ was the union of two separate rat brain hemispheres which, for convenience, were embedded in an agar ball. In order to obtain non invasive Cavalieri transects, the ball was submitted - after random rotation - to magnetic resonance scanning. With the idea of reducing the error variance, the two hemispheres were arranged relatively close and perpendicular to each other inside the agar ball. This was considered plausible by virtue of the result given in Section A model for the antithetic effect below. But then an apparent paradox arises because the variance predictor depends on $S$ and $T$ only, and not on the shape, nor on the spatial arrangement of the separate parts constituting the set $Y$. This point was not discussed in the latter paper, and our purpose is to explore it here.

It may be conjectured that, for each $T$, the true variance of the ICav volume estimator will vary less among isotropic directions when the brain hemispheres are mutually perpendicular, than when they are aligned. The mean variance over isotropic directions, however, should be nearly the same for any arrangement. Our Monte Carlo results confirm these conjectures for the material studied.

\section{THE ICAV DESIGN: THEORY AND PRELIMINARY CONCLUSIONS}

Fix an orthogonal reference frame $O x_{1} x_{2} x_{3}$. An axis $L_{1}(0, u)$ of direction $u$ is obtained by joining the origin $O$ with a point $u \in \mathbb{S}_{+}^{2}$ on the unit hemisphere. The direction $u$ is parametrized by its spherical polar coordinates $(\phi, \theta)$, where $\phi \in[0,2 \pi), \theta \in[0, \pi / 2]$ represent the longitude and the colatitude angles respectively, see Fig. 1a. 


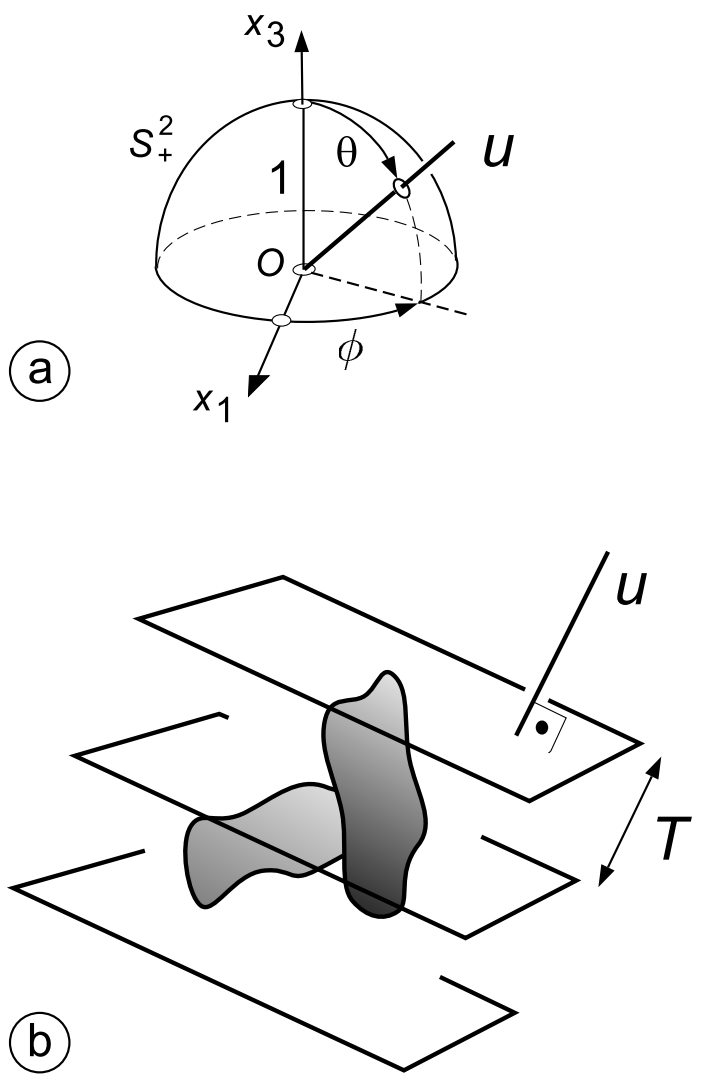

Fig. 1. (a) Axial direction $u$ through a point of spherical polar coordinates $(\phi, \theta)$ on the unit hemisphere. (b) An isotropic Cavalieri series of planes normal to an isotropic axis $u$, hitting an object.

A plane normal to the axis $L_{1}(0, u)$ at a distance $p \in \mathbb{R}$ from $O$ is denoted by $L_{2}(p, u)$. An isotropic Cavalieri series of planes a constant distance $T>0$ apart, see Fig. 1b, may be generated in the following two steps.

1. Generate an isotropic sampling axis of direction $u=(\phi, \theta)$ as follows:

$$
\phi=2 \pi U_{1}, \quad \theta=\cos ^{-1}\left(U_{2}\right),
$$

where $U_{1}, U_{2}$ are two independent uniform random (UR) numbers in the interval $[0,1)$. The pertinent probability elements are,

$$
\mathbb{P}(\mathrm{d} u)=\frac{\mathrm{d} u}{2 \pi}, \quad u \in \mathbb{S}_{+}^{2},
$$

or,

$$
\mathbb{P}(\mathrm{d} \phi, \mathrm{d} \theta)=\frac{\sin \theta \mathrm{d} \phi \mathrm{d} \theta}{2 \pi}
$$

2. For each pair $(\phi, \theta)$, the UR offset $z$ in the interval $[0, T)$ for the Cavalieri series may be generated by taking a third independent UR number $U_{3}$ and setting:

$$
z=U_{3} T
$$

Thus,

$$
\mathbb{P}(\mathrm{d} z)=\frac{\mathrm{d} z}{T}, \quad z \in[0, T) .
$$

The isotropic Cavalieri series of planes may now be represented as follows,

$$
\Lambda_{z, u}=\left\{L_{2}(z+k T, u), k \in \mathbb{Z}\right\},
$$

with the joint probability element,

$$
\mathbb{P}(\mathrm{d} u, \mathrm{~d} z)=\mathbb{P}(\mathrm{d} u) \cdot \mathbb{P}(\mathrm{d} z) .
$$

The area of a planar section $Y \cap L_{2}(p, u)$ is denoted by $A(p, u)$. If $Y \cap L_{2}(p, u)=\emptyset$, then we set $A(p, u)=0$. Suppose that the set $Y$ is intersected by a isotropic Cavalieri series. Then the ICav estimator

$$
\widehat{V} \equiv \widehat{V}(z, u)=T \sum_{k \in \mathbb{Z}} A(z+k T, u)
$$

is an unbiased estimator (UE) of $V$, that is,

$$
\mathbb{E}(\widehat{V})=\mathbb{E}_{u}\left\{\mathbb{E}_{z}(\widehat{V} \mid u)\right\}=\mathbb{E}_{u}(V)=V .
$$

The subscripts $u, z$ indicate expectations with respect to $\mathbb{P}(\mathrm{d} u)$ and $\mathbb{P}(\mathrm{d} z)$, respectively. Recall that the identity $\mathbb{E}_{z}(\widehat{V} \mid u)=V$ expresses the unbiasedness of the ordinary Cavalieri estimator for any cutting direction $u \in \mathbb{S}_{+}^{2}$.

Remarks on notation. In the sequel, true variances will be denoted by $\operatorname{Var}(\cdot)$, whereas their predictors, or estimators, will be denoted by $\operatorname{var}(\cdot)$. For a positive random variable the square coefficient of variation is $\mathrm{CV}^{2}(\cdot)=\operatorname{Var}(\cdot) /\{\mathbb{E}(\cdot)\}^{2}$. If the random variable is an estimator, then the equivalent notation $\mathrm{CE}^{2}(\cdot)$, called the square coefficient of error, is used.

An approximation of the variance of the ICav volume estimator - based on G. Matheron"s transitive theory - is,

$$
\operatorname{var}_{I C a v}\{\widehat{V}(z, u)\}=\frac{\pi}{360} S T^{4}
$$

which represents the 'extension term' of the true variance, namely a sort of mean trend which excludes the typical oscillations of the true variance caused by the so called 'Zitterbewegung' effect. For a complete derivation of the preceding result, and early references, see Cruz-Orive (2013).

On the other hand, the true variance of the ICav estimator $\widehat{V} \equiv \widehat{V}(z, u)$ may be expressed as follows,

$$
\begin{aligned}
\operatorname{Var}(\widehat{V}) & =\operatorname{Var}_{u}\left\{\mathbb{E}_{z}(\widehat{V} \mid u)\right\}+\mathbb{E}_{u}\left\{\operatorname{Var}_{z}(\widehat{V} \mid u)\right\} \\
& =\operatorname{Var}_{u}(V)+\mathbb{E}_{u}\left\{\operatorname{Var}_{z}(\widehat{V} \mid u)\right\} \\
& =\mathbb{E}_{u}\left\{\operatorname{Var}_{z}(\widehat{V} \mid u)\right\}
\end{aligned}
$$


namely the mean over isotropic directions of the variance of $\widehat{V}$ conditional on a given direction; the latter is the variance of the ordinary Cavalieri estimator of $V$ for a given direction.

In this study we have addressed and answered the following questions for the material studied.

1. Is the right hand side (rhs) of Eq. 10 a reliable predictor of the rhs of Eq. 11? We checked this empirically by automatic Monte Carlo resampling of $\widehat{V}$ (details in the corresponding section below) on the two different object arrangements illustrated in Fig. 2. The results displayed in Fig. 4a,b, (red lines), show that Eq. 10 is a reasonable variance predictor, at least when the mean number of Cavalieri transects is no less than about 1.5. This is consistent with the fact that the plots (black curves) of $\operatorname{Var}(\widehat{V})$ versus the period $T$ vary relatively very little among the two object arrangements.

2. If Eq. 10 is a reasonable variance predictor no matter the arrangement of the parts constituting the object, then what do we gain by choosing an arrangement like the one in Fig. 2a, say, which should in principle produce an antithetic effect? The answer is twofold. First, the horizontal axes in Fig. 4a,b reveal that, for a given period $T$, the mean number of sections for object $Y_{2}$ is about $25 \%$ greater than for object $Y_{1}$. And second, Fig. 5 shows that, for a given period $T$, it is $\operatorname{Var}_{u}\left\{\operatorname{Var}_{z}(\widehat{V} \mid u)\right\}$, and not $\mathbb{E}_{u}\left\{\operatorname{Var}_{z}(\widehat{V} \mid u)\right\}=\operatorname{Var}(\widehat{V})$, that is reduced by choosing the arrangement in Fig. 2a. In Fig. 4a,b it can already be appreciated that the spread of $\operatorname{Var}_{z}(\widehat{V} \mid u)$ for varying $u$ is greater for the object arrangement in Fig. 2b than for the one in Fig. 2a. Thus, the arrangement of the objects studied here affects the mean number of Cavalieri sections on the one hand, and the stability of the conditional variance $\operatorname{Var}_{z}(\widehat{V} \mid u)$ among isotropic directions on the other, but its mean $\operatorname{Var}(\widehat{V})$ is almost unaffected.

3. Suppose that, instead of Eq. 10, we use for each sectioning direction $u$ the classical predictor given by Eq. 12 below for the ordinary Cavalieri estimator. What is the performance of the latter predictor? Fig. 4c,d suggest that the classical predictor is generally poorer than the ICav predictor, at least in the mean over isotropic directions (its dispersion is not shown). The dependence of this predictor on the smoothness constant $q \in[0.1]$ is also an important snag, not only because it generally depends on the cutting direction, but also because it cannot be reliably estimated. Among the three values considered, the better performance corresponded to $q=0.42$, when $\alpha(0.42) \approx 1 / 36$, see below.

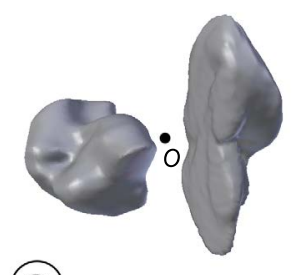

(a) (b)

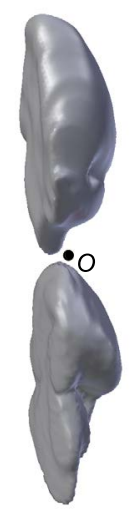

Fig. 2. Computer reconstructions of the two test objects studied. (a) Object $Y_{1}$ with the two rat brain hemispheres placed in antithetic position. (b) Object $Y_{2}$ with longitudinally arranged hemispheres. See Section Test objects.

The classical variance predictor for the ordinary Cavalieri volume estimator conditional on a given cutting direction $u$, reads as follows,

$$
\begin{aligned}
\operatorname{var}_{\text {Cav }}(\widehat{V} \mid u) & =\alpha(q) \cdot\left(3 C_{0}-4 C_{1}+C_{2}\right) T^{2}, \\
C_{i} & =\sum_{k \in \mathbb{Z}} f_{k} \cdot f_{k+i}, \quad i=0,1,2, \\
f_{k} & =A(z+k T, u \mid u),
\end{aligned}
$$

which is also due to G. Matheron - pertinent references are given in the Introduction. The numerical coefficient $\alpha(q)$ depends on the smoothness constant $q \in[0,1]$ of the area function $A(p, u)$, namely the (possibly fractional) order of the first non-continuous derivative of $A(p, u)$ for a given $u$. The value of $\alpha(q)$ ranges between $\alpha(0)=1 / 12$ and $\alpha(1)=1 / 240$. The available estimator of $q$ is not particularly robust, see García-Fiñana and Cruz-Orive (2004). For human brain, empirical studies have shown that $\alpha(0.42) \approx$ $1 / 36$ yields reasonable variance predictions, see the latter paper and also Cruz-Orive (2006) and CruzOrive et al. (2014).

The rest of the paper is devoted to the details and results that support the foregoing conclusions.

\section{MATERIAL}

\section{TEST OBJECTS}

The basic material consisted of computer reconstructions of the two hemispheres of a rat brain 
obtained with the aid of the 3D modelling software Blender, (http://www.blender.org/). The input was an exhaustive series of MRI, $1 \mathrm{~mm}$ thick virtual slices obtained at $3 \mathrm{~T}$ resolution. Each subset boundary was thereby approximated by a closed, smoothened triangulated mesh. For a description of the image processing steps followed see González-Villa et al. (2017).

The study was carried out on two test objects $Y_{1}$ and $Y_{2}$ shown in Fig. 2a,b, respectively. The object $Y_{1}$ was the union of both hemispheres arranged with their long axes approximately perpendicular to each other, and with their nearly flat faces facing each other. The straight line segment joining the hemisphere centroids was approximately normal to either longitudinal axis. On the other hand, for the object $Y_{2}$ the hemispheres were arranged with their long axes approximately collinear, and with their nearly flat faces approximately coplanar.

\section{A MODEL FOR THE ANTITHETIC EFFECT}

The true variance of the ICav estimator of volume depends on the variability of the number of Cavalieri transects (or, equivalently, on the variability of the caliper length among directions) and on the variability of the transect areas for each direction. We propose a simple model for the former contribution - section areas are not involved in the model. The goal is to see whether or not arranging the brain hemispheres as in the object $Y_{1}$ above implies some statistical advantage.

As a simple model for the longest axes of the two brain hemispheres constituting the object $Y_{1}$, consider an X-shaped object $X_{\alpha} \in \mathbb{R}^{3}$ consisting of two straight line segments or 'rods' of length 2 , intersecting at their midpoint $O$, making an angle $2 \alpha \in[0, \pi / 2]$, and lying on the $O x_{2} x_{3}$ plane of an orthogonal reference frame $O x_{1} x_{2} x_{3}$, see Fig. 1a and Fig. 3. The problem is to find a value of $\alpha$ such that $\operatorname{Var}_{u}\left\{2 H_{\alpha}(u)\right\}$ is minimal, where $2 H_{\alpha}(u)$ denotes the length of the orthogonal linear projection of $X_{\alpha}$ onto a isotropic axis of direction $u \in \mathbb{S}_{+}^{2}$, that is the caliper length of the object along $u$. The function $H_{\alpha}(u)$, is in fact the support function of $X_{\alpha}$ with origin $O$, namely the dot product of the vector $(0, \sin \alpha, \cos \alpha)$ times the vector $u=(\sin \theta \cos \phi, \sin \theta \sin \phi, \cos \theta)$. Thus,

$$
H_{\alpha}(u)=\sin \theta \sin \phi \sin \alpha+\cos \theta \cos \alpha .
$$

Now, using the probability element given by Eq. 3, the mean half caliper length is,

$$
\mathbb{E}_{u}\left\{H_{\alpha}(u)\right\}=\frac{1}{2} \sqrt{1+\sin (2 \alpha)},
$$

which depends on the shape factor $\alpha$. Its minimum value is $1 / 2$ for $\alpha=0$, when the object degenerates into a single rod, and its maximum is $\sqrt{2} / 2$ for $\alpha=\pi / 4$, when the object is a 'Greek cross' ' + '.

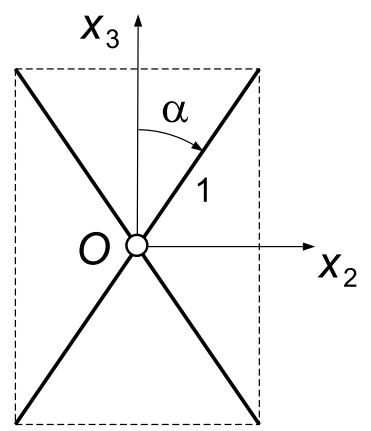

Fig. 3. X-shaped model object (heavy diagonal lines).

On the other hand, the variance of the half caliper length is,

$$
\operatorname{Var}_{u}\left\{H_{\alpha}(u)\right\}=\frac{1}{12}-\frac{3 \pi-8}{12 \pi} \sin (2 \alpha),
$$

whose minimum value is equal to $(4-\pi) /(6 \pi)$ when $\alpha=\pi / 4$, namely when the object is a Greek cross. Thus, for a given period $T$ the brain arrangement $Y_{1}$ is likely to exhibit a smaller variaton in the number of Cavalieri sections among orientations, than other patterns.

For object $Y_{2}$ a corresponding model consists of two aligned rods, namely of a single rod of length 4 with origin at its midpoint. Here $H(u)=2 \cos (\theta)$, so that $\mathbb{E}_{u}\{H(u)\}=1$, which is about $41 \%$ greater than the value $\sqrt{2} / 2$ corresponding to the Greek cross model for $Y_{1}$. On the other hand, $\operatorname{Var}_{u}\{H(u)\}=1 / 3$, which is about 6.32 times the Greek cross value of $(4-\pi) /(6 \pi) \approx 0.0455$.

\section{MONTE CARLO EXPERIMENT}

\section{PROBE GENERATION}

An orthogonal reference frame $O x_{1} x_{2} x_{3}$ was adopted for either test object with the origin $O$ at the midpoint of the straight line segment joining the two hemisphere centroids. The polar axis $\mathrm{Ox}_{3}$ was parallel to the long axis of a hemisphere. For a proper sampling, a reference ball $D$ was adopted. This ball was centred at $O$, and it was the smallest ball enclosing the union of both test objects. The actual diameter of $D$ was $2 R=6.13 \mathrm{~cm}$ for either arrangement $Y_{1}$ or $Y_{2}$. (The longest axis of each rat brain hemisphere did not exceed $2.5 \mathrm{~cm}$ in length.)

The protocol described next was applied to each test object. 
Axial isotropic directions. The first step was to generate a total of $M=N_{1} N_{2}$ axial directions

$$
\left\{u_{i j}, i=0,1, \ldots, N_{1}-1, j=0,1, \ldots, N_{2}-1\right\}
$$

following a systematic protocol, namely,

$$
\begin{aligned}
\phi_{i} & =\left(U_{1}+i\right) \cdot\left(2 \pi / N_{1}\right), \\
\theta_{j} & =\cos ^{-1}\left[\left(U_{2}+j\right) / N_{2}\right],
\end{aligned}
$$

where $\left(U_{1}, U_{2}\right)$ are as defined for Eq. 1 and Eq. 2, respectively. Here we chose $N_{1}=50$ and $N_{2}=25$, hence $M=1250$ directions. For ease of notation, henceforth we rearrange the matrix $\left\{u_{i j}\right\}$ row-wise into a vector of length $M$, namely,

$$
\left(u_{i}, i=1,2, \ldots, M\right) \text {. }
$$

Cavalieri sections for each direction. Within the interval $[-R, R]$ corresponding to the diameter of the reference ball, a total of $N$ basic abscissas were generated a small gap $\Delta=2 R / N$ apart, namely,

$$
\left\{p_{r}=-R+(U+r) \Delta, r=0,1, \ldots, N-1\right\},
$$

where $U$ is a third, independent UR number in the interval $[0,1)$. Then, a 'databank' of $N$ section areas, namely,

$$
\left\{A\left(p_{r}, u_{i}\right), r=0,1, \ldots, N-1\right\},
$$

were computed automatically for each direction $u_{i}$ with the aid of the bisect method from the bpy library of the Blender software. Thus, we computed a total of $M$ databanks. For each direction $u_{i}$, the corresponding databank facilitated the generation of Cavalieri samples for $N$ different periods, namely for

$$
\left\{T_{k}=k \Delta, k=1, \ldots, N\right\} .
$$

In this study we adopted $N=2000$, so that the subsequent graphs of the empirical curves would look continuous to the eye. With period $T \equiv T_{k}$, a total of $k$ Cavalieri samples are available for each direction. The abscissas corresponding to the $s$ th sample, $s=$ $1,2, \ldots, k$, were

$$
\left\{p_{s j}=-R+s \Delta+j T, j=1,2, \ldots, J\right\},
$$

where $J=\lfloor 2 R /(T+1)\rfloor$. The corresponding Cavalieri section areas,

$$
\left\{A\left(p_{s j}, u_{i}\right), j=1,2, \ldots, J\right\},
$$

were extracted directly from the $i$ th databank. It should be borne in mind that Cavalieri samples with all section areas equal to zero should be retained for the subsequent calculations. In other words, for large $T$ it is possible that the ICav volume estimator is equal to zero, and this affects the mean and variance of the estimator. The mean of the number $n(u)$ of Cavalieri sections hitting an object along a direction $u$ is

$$
\mathbb{E}\{n(u)\}=H(u) / T,
$$

where $H(u)$ is as defined above, namely the caliper length of the object along the direction $u$. Note that $\mathbb{E}\{n(u)\}$ may be less than 1 .

\section{EMPIRICAL MEANS AND VARIANCES}

For a given period $T \equiv T_{k}, k \in\{1,2, \ldots, N\}$, the following computations were carried out.

For a given direction $u_{i}$, the empirical version of $\widehat{V}(z, u)$ from the sth Cavalieri sample is,

$$
\widehat{V}_{s i}=T \sum_{j=1}^{J} A\left(p_{s j}, u_{i}\right), \quad s=1,2, \ldots, k,
$$

and its mean over the $k$ samples,

$$
\mathbb{E}_{e}\left(\widehat{V}_{i}\right)=\frac{1}{k} \sum_{s=1}^{k} \widehat{V}_{s i}, \quad i=1,2, \ldots, M,
$$

is the empirical version of $\mathbb{E}_{z}(\widehat{V} \mid u)$. Henceforth the subscript ' $e$ ' refers to 'empirical', namely to a Monte Carlo estimator with (usually) negligible error. The mean over directions,

$$
\widehat{V}=\mathbb{E}_{e}\left\{\mathbb{E}_{e}\left(\widehat{V}_{i}\right)\right\}=\frac{1}{M} \sum_{i=1}^{M} \mathbb{E}_{e}\left(\widehat{V}_{i}\right)
$$

corresponds to $\mathbb{E}_{u}\left\{\mathbb{E}_{z}(\widehat{V} \mid u)\right\}=\mathbb{E}_{u}(V)=V$. Further,

$$
\operatorname{Var}_{e}\left(\widehat{V}_{i}\right)=\frac{1}{k} \sum_{s=1}^{k}\left[\widehat{V}_{s i}-\mathbb{E}_{e}\left(\widehat{V}_{i}\right)\right]^{2},
$$

is the emprical version of $\operatorname{Var}_{z}(\widehat{V} \mid u)$. Its mean over directions,

$$
\operatorname{Var}_{e}(\widehat{V})=\mathbb{E}_{e}\left\{\operatorname{Var}_{e}\left(\widehat{V}_{i}\right)\right\}=\frac{1}{M} \sum_{i=1}^{M} \operatorname{Var}_{e}\left(\widehat{V}_{i}\right)
$$

is the empirical version of $\mathbb{E}_{u}\left\{\operatorname{Var}_{z}(\widehat{V} \mid u)\right\}=\operatorname{Var}(\widehat{V})$. Finally,

$$
\operatorname{Var}_{e}\left\{\operatorname{Var}_{e}\left(\widehat{V}_{i}\right)\right\}=\frac{1}{M} \sum_{i=1}^{M}\left[\operatorname{Var}_{e}\left(\widehat{V}_{i}\right)-\operatorname{Var}_{e}(\widehat{V})\right]^{2},
$$

is the empirical version of $\operatorname{Var}_{u}\left\{\operatorname{Var}_{z}(\widehat{V} \mid u)\right\}$, whose behaviour we wanted to compare among both test objects. 

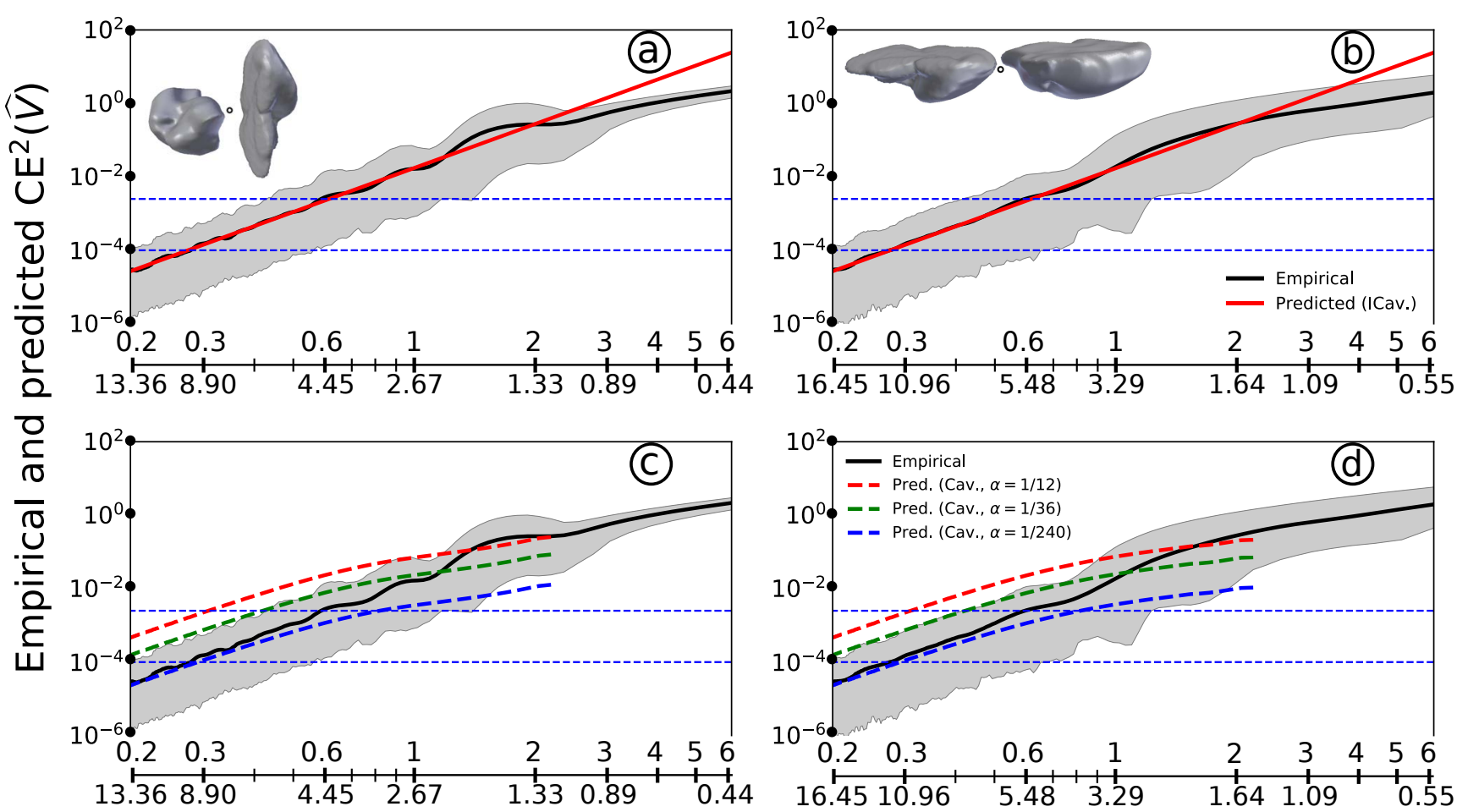

\section{$T, \mathrm{~cm}$, (above axis) and average number of sections among directions}

Fig. 4. (a,b) Empirical $\mathrm{CE}_{e}^{2}(\widehat{V})$ and predicted $\mathrm{ce}_{\mathrm{ICav}}^{2}(\widehat{V})$ square coefficients of error of the ICav volume estimator for each of the two objects studied, as functions of the sampling period T. The grey bands enclose the Monte Carlo realizations of the conditional square coefficient of error $\operatorname{CE}_{z}^{2}(\widehat{V} \mid u)$ for 1250 directions $u$, as a function of $T$. (c, d) Behaviour of the classical Cavalieri predictors (broken lines) for each object, (Eq. 33 divided by $V^{2}$ ), as functions of $T$. In the horizontal axes, for each T the mean number of sections is the mean of the rhs of Eq. 25 over orientations. In each panel, the two dotted, horizontal lines, correspond to coefficients of error of 0.01 and 0.05 respectively.

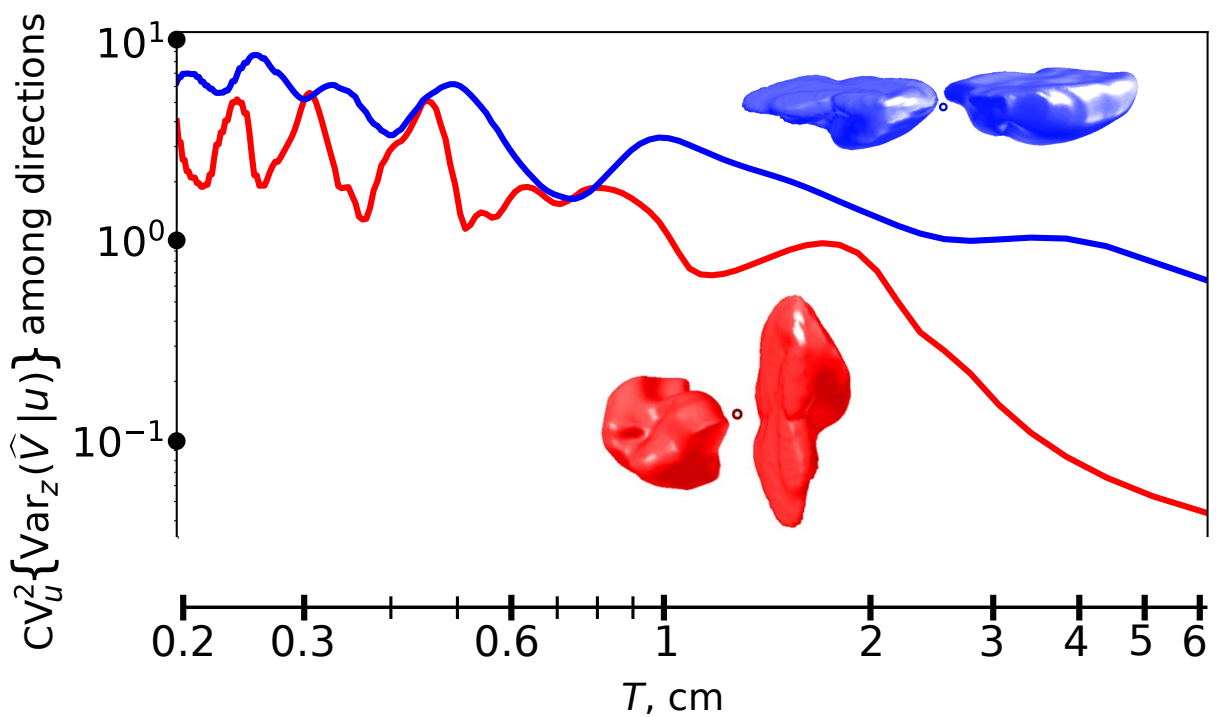

Fig. 5. Behaviour of the square coefficient of variation $\mathrm{CV}_{u}^{2}\left\{\operatorname{Var}_{z}(\widehat{V} \mid u)\right\}$ of the conditional Cavalieri variance for a given orientation, among isotropic orientations. Here the antithetic arrangement, (object $Y_{1}$, in red), shows an advantage over $Y_{2}$, (in blue). 


\section{VARIANCE PREDICTORS}

For each period $T \equiv T_{k}, \quad k=1,2, \ldots, N$, the ICav variance predictor was computed directly from Eq. 10 because the surface area of the right and left hemisphere reconstructions was known, namely $7.682 \mathrm{~cm}^{2}$ and $8.340 \mathrm{~cm}^{2}$, respectively. Thus $S=$ $16.022 \mathrm{~cm}^{2}$, see González-Villa et al. (2017), p. 127.

For each orientation $u \equiv u_{i}$, and each Cavalieri sample, the classical predictor $\operatorname{var}_{\text {Cav }}\left(\widehat{V}_{s i}\right)$ was computed with Eq. 12. Let $n_{s i}$ denote the number of Cavalieri sections hitting the object. Then, for each $\alpha=1 / 12,1 / 36,1 / 240$ we computed,

$$
\begin{aligned}
\operatorname{var}_{C a v}\left(\widehat{V}_{s i}\right) & =\alpha T^{2}\left(3 C_{0}-4 C_{1}+C_{2}\right), \quad n_{s i} \geq 3 \\
\operatorname{var}_{C a v}\left(\widehat{V}_{s i}\right) & =\alpha T^{2}\left(C_{0}-C_{1}\right), \quad n_{s i}=1,2 \\
C_{h} & =\sum_{j=1}^{n_{s i}-h} f_{j} \cdot f_{j+h}, \quad h=0,1,2 \\
f_{j} & =A\left(p_{s j}, u_{i}\right) .
\end{aligned}
$$

Finally, for each $T$ the overall mean,

$$
\operatorname{var}_{\mathrm{Cav}}(\widehat{V})=\frac{1}{M N} \sum_{i=1}^{M} \sum_{s=1}^{k} \operatorname{var}_{\mathrm{Cav}}\left(\widehat{V}_{s i}\right),
$$

is the classical predictor of $\operatorname{Var}(\widehat{V})$, alternative to Eq. 10.

\section{RESULTS}

Prior to plotting them in Fig. 4, empirical and predicted variances were divided by $V^{2}$ to obtain the corresponding square coefficients of error. The volumes of the right and left hemisphere reconstructions, and hence of their union, were known, namely $1.419,1.437$ and $V=2.856 \mathrm{~cm}^{3}$, respectively, see González-Villa et al. (2017), p. 127.

In Fig. 4a,b, the empirical square coefficient of error $\mathrm{CE}_{e}^{2}(\widehat{V})$, computed via Eq. 30 for each period $T$, is plotted as a black continuous curve, whereas the corresponding ICav predictor $\mathrm{ce}_{\mathrm{ICav}}^{2}(\widehat{V})$, computed via Eq. 10, is plotted as a red line.

In the same two panels, for each value of $T$ the grey band contains all the 1250 realizations of the empirical square coefficient of error $\mathrm{CE}_{e}^{2}\left(\widehat{V}_{i}\right)$, conditional on each of the 1250 directions considered, and computed via Eq. 29.

In Fig. 4c,d, the classical ce $\mathrm{Cav}_{\mathrm{Cav}}(\widehat{V})$, computed via Eq. 33, is plotted against the period $T$ by means of broken lines, with the meaning of each colour explained in the inset of Fig. 4d.
Finally, for each of the two objects the curves in Fig. 5 represent the rhs of Eq. 31 divided by the square of the rhs of Eq. 30 to obtain the square coefficient of variation of $\operatorname{Var}_{e}\left(\widehat{V}_{i}\right)$ among directions.

\section{DISCUSSION AND CONCLUSIONS}

The preceding results confirm the preliminary conclusions given in the second section for the concrete geometrical objects studied. For the present purpose, the closeness of the smoothened triangulations used in the reconstructions, to the actual biological objects, was of no concern. It seems unlikely that the use of other objects and arrangements would affect the main conclusions.

Apart from its good performance, the error variance predictor given by Eq. 10 is much easier to compute than the classical Cavalieri predictor given by Eq. 12. In practice, a UE of $S$ is available from the data, namely,

$$
\widehat{S} \equiv \widehat{S}(z, u)=\frac{4}{\pi} T \sum_{k \in \mathbb{Z}} B(z+k T, u),
$$

where $B(\cdot)$ denotes transect boundary length, see CruzOrive et al. (2010) for further details.

With reference to Fig. $4 a, b$, the fact that the true error variance $\operatorname{Var}(\widehat{V})$, (black curves), is almost insensitive to object shape as a function of $T$ sounds paradoxical at the first sight, but it should be borne in mind that this variance is an average over isotropic directions, which is a strong condition. This is consistent with the fact that the variance predictor given by Eq. 10, (red lines), is fairly accurate, and it only depends on $S$ and $T$, not on object shape.

The reduction in the mean number of Cavalieri sections, and in the variability of $\operatorname{Var}_{z}(\widehat{V} \mid u)$, however, should justify the arrangement of the subsets constituting the target object into a favourable position. The simple models studied confirm that the arrangement of two elongated subsets into a rectangular cross induces a reduction in the mean and the variance of the number of Cavalieri sections for a given period.

We have not carried out a similar study for the ICav estimator $\widehat{S}(z, u)$, first to avoid distraction from our main goal, and second because no error variance predictor analogous to $\operatorname{var}_{\text {ICav }}\{\widehat{V}(z, u)\}$ exists which is both general and easy to compute. The conclusions relative to $\operatorname{Var}_{z}(\widehat{S} \mid u)$ are unlikely to differ much, however, because, for a given object, transect area and 
perimeter length tend to be fairly strongly correlated. This statement is based on the fact that the ratio $B / \sqrt{A}$ (involved in the predictor of the error variance of the point counting estimator of planar area) tends to be rather stable for a given type of transects, (Gundersen and Jensen, 1987).

\section{ACKNOWLEDGMENTS}

The authors acknowledge financial support from the Spanish project AYA2015-66357-R (MINECO/FEDER).

\section{REFERENCES}

Cruz-Orive, LM (2006). A general variance predictor for Cavalieri slices. J Microsc 222:158-65.

Cruz-Orive, LM (2013). Variance predictors for isotropic geometric sampling, with applications in forestry. Statist Methods Appl 22:3-31.
Cruz-Orive, LM, Gelšvartas, J, Roberts, N (2014). Sampling theory and automated simulations for vertical sections, applied to human brain. J Microsc 253:119-50.

Cruz-Orive, LM, Ramos-Herrera, ML, Artacho-Pérula, E (2010). Stereology of isolated objects with the invariator. J Microsc 240:94-110.

García-Fiñana, M, Cruz-Orive, LM (2004). Improved variance prediction for systematic sampling on $\mathbb{R}$. Statistics 38:243-72.

González-Villa J, Cruz M, Cruz-Orive LM (2017). On the precision of the nucleator. Image Anal Stereol 36:12334.

Gundersen, HJG, Jensen, EB (1987). The efficiency of systematic sampling in stereology and its prediction. $\mathrm{J}$ Microsc 147:229-63.

Kiêu, K, Souchet, S, Istas, J (1999). Precision of systematic sampling and transitive methods. J Statist Plan Inf 77:263-79. 